\title{
Idiopathic Pulmonary Hemosiderosis Associated with Emphysematous Change in an Adult Who Underwent Lung Transplantation
}

\author{
Kyoko Gocho ${ }^{1}$, Kenya Sato ${ }^{1}$, Keisuke Imasaka ${ }^{1}$, Nobuyuki Hamanaka ${ }^{1}$, Miki Takahashi ${ }^{1}$, \\ Kunihiko Shimizu ${ }^{1}$ and Tamiko Takemura ${ }^{2}$
}

\begin{abstract}
:
Idiopathic pulmonary hemosiderosis is characterized by repeated alveolar hemorrhaging. We herein report a 52-year-old Japanese woman who had shortness of breath, diffuse small nodules, thin-walled cysts, and bronchiolectasis. A surgical lung biopsy revealed peribronchial hemosiderosis, centrilobular emphysema, and fragile elastic fibers of the alveolar septa and small vessels. She ultimately underwent living-donor lung transplantation five years after the first visit.
\end{abstract}

Key words: idiopathic pulmonary hemosiderosis, peribronchiol hemosiderosis, emphysematous change, Ehlers-Danlos syndrome, lung transplantation

(Intern Med 60: 117-122, 2021)

(DOI: 10.2169/internalmedicine.5142-20)

\section{Introduction}

Idiopathic pulmonary hemosiderosis (IPH) is characterized by repeated alveolar hemorrhaging and deposition of hemosiderin in the lung (1). IPH commonly develops in childhood and presents with the classic triad of irondeficiency anemia, hemoptysis, and infiltration on chest images (2).

The deposition of hemosiderin and hemosiderin-laden macrophages in the lung parenchyma and interstitium is the most typical histopathological finding, indicating repeated alveolar hemorrhaging (3). Furthermore, the existence of vascular malformations, malignancy, pulmonary vasculitis/ capillaritis, granulomatous inflammation, or infectious agents can help exclude IPH (4). IPH is usually diagnosed based on typical clinical symptoms and histopathological findings using small specimens of a transbronchial lung biopsy and/or bronchoalveolar lavage $(3,5)$.

We herein report a rare case of IPH in an adult patient who eventually underwent living-donor lung transplantation. The patient was examined through long-term observational chest computed tomography (CT) and detailed histopathological findings.

\section{Case Report}

A 52-year-old Japanese woman was admitted to our hospital with complaints of progressive dyspnea for the past 4 years. She was a non-smoker and did not have any marked respiratory medical history. In addition, she did not have food allergies or a family history of genetic disease.

At the initial visit, she had a lean body with a low body mass index $\left(16.9 \mathrm{~kg} / \mathrm{m}^{2}\right)$. She had a temperature of $36.0^{\circ} \mathrm{C}$, pulse rate of 74 beats per min, blood pressure of 136/80 $\mathrm{mmHg}$, and oxygen saturation of $95 \%$ on room air. Chest auscultation did not reveal crackles in the lung fields or murmur in the heart. Skin, joint, and skeletal abnormalities were not evident.

A laboratory evaluation yielded normal results. She had no anemia (hemoglobin $14.3 \mathrm{~g} / \mathrm{dL}$ ), and inflammatory factors, autoimmune markers (i.e., antinuclear antibody and various specific antibodies including antineutrophilic cytoplasmic antibody), Krebs von den Lungen-6, and surfactant

${ }^{1}$ Department of Respiratory Medicine, Saiseikai Yokohamashi Tobu Hospital, Japan and ${ }^{2}$ Department of Pathology, Kanagawa Cardiovascular and Respiratory Center, Japan

Received: April 21, 2020; Accepted: July 6, 2020; Advance Publication by J-STAGE: August 22, 2020

Correspondence to Dr. Kyoko Gocho, kyokogocho@yahoo.co.jp 

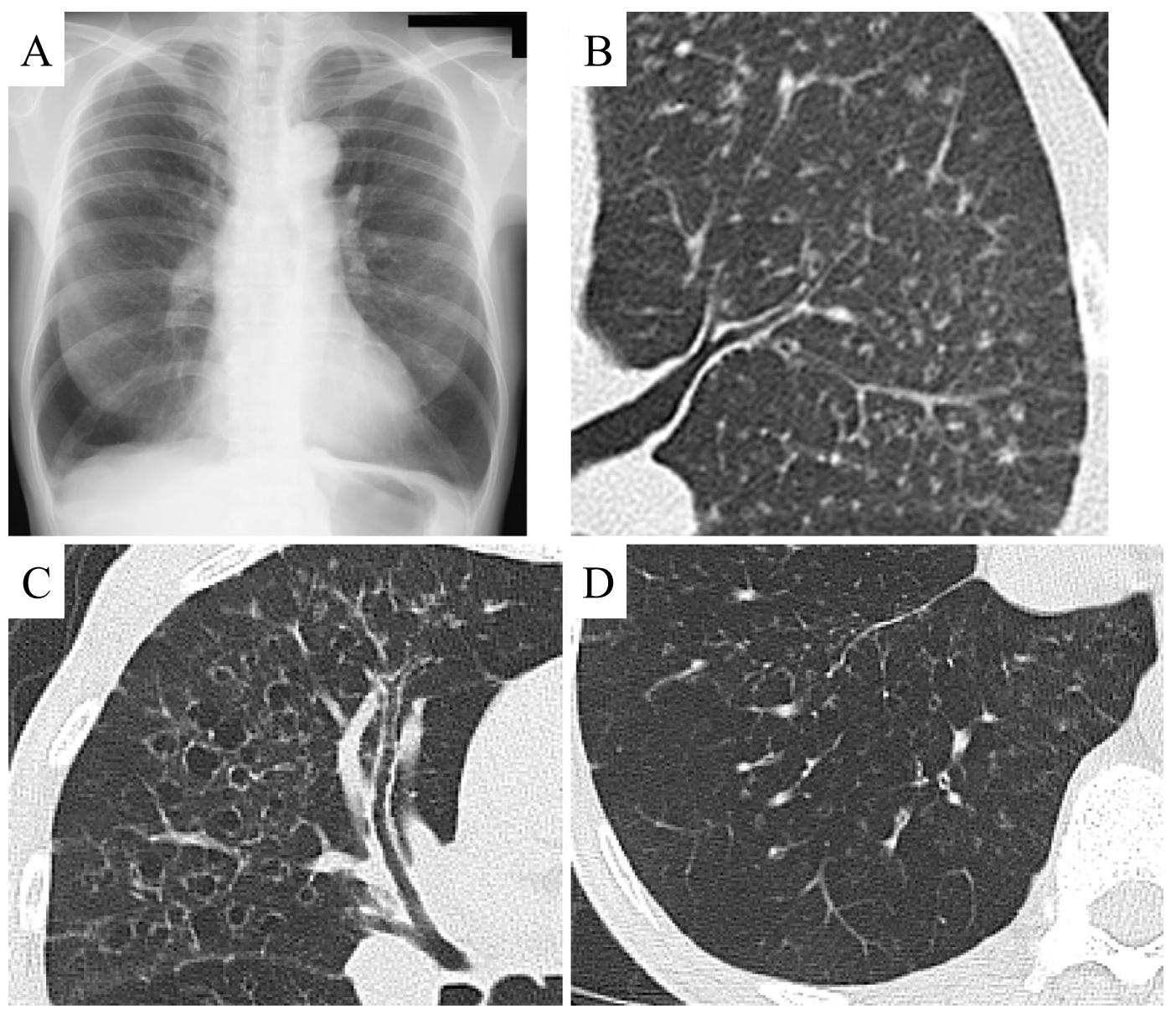

Figure 1. (A) Chest radiography showed diffuse reticular shadows in the bilateral upper lung fields and hyperpermeability in the lower lung fields. $(\mathrm{B}, \mathrm{C})$ Chest computed tomography showed centrilobular distribution of diffuse small nodules, emphysematous change, and thickened bronchioli in the bilateral upper and middle lobes. (D) Thickened bronchioli with hyperinflation of the lung in lower lobe.

protein-D were not elevated.

Chest radiography showed diffuse reticular shadows in the bilateral upper lung fields and hyperpermeability in the lower lung fields (Fig. 1A). Chest CT showed centrilobular distribution of diffuse small nodules with emphysematous change and thickened bronchioli in the bilateral upper and middle lobes (Fig. 1B, C). In the lower lobes, thickened bronchioli with hyperinflation of the lung were revealed (Fig. 1D).

The pulmonary function test revealed mixed ventilatory impairment (vital capacity: $1.57 \mathrm{~L}, 63.1 \%$ predicted; forced expiratory volume in 1 second as the percentage of forced vital capacity: $48.8 \%$ ), with almost within-the-normal-range levels of diffusing capacity for carbon monoxide. Elevated values for residual volume (RV) and ratio of $\mathrm{RV}$ to total lung capacity (TLC) were also revealed (RV: $1.82 \mathrm{~L}, \mathrm{RV} /$ TLC: $53.2 \%$ ) (Table 1).

We performed video-assisted thoracoscopic surgery (VATS) to reach a definitive diagnosis three years after the initial visit. The specimen obtained through VATS showed emphysematous change with raptured elastic fibers, in addition to deposition of hemosiderin and hemosiderin-laden macrophages in the peribronchial alveolar space, alveolar septum, and vessel walls (Fig. 2A-C). The respiratory bronchioles associated with the hemosiderosis lesion were narrowed, while some terminal bronchioles were dilated with the accumulation of mucus and lymphocyte infiltration into the mucosa (Fig. 2B). Hemosiderin and foreign body giant cells were deposited in the vessel walls, while elastic fibers of the vessel wall of the same lesion were obscured. However, vasculitis was not detected (Fig. 2C, D).

Vascular-type Ehlers-Danlos syndrome was considered as a differential diagnosis because of the vulnerability of elastic fibers in the alveolar septum and blood vessel walls. However, the patient did not exhibit clinical extra-pulmonary abnormality (e.g., skin, joints, and anatomy) and did not have specific family history. There was no evidence of any abnormality in the electrophoretic pattern or the ability to produce type III collagen using cultured skin fibroblasts obtained from a skin biopsy. Of note, electron microscopy did not reveal an abnormality of collagen fibrils. Based on this evidence, the patient was diagnosed with IPH.

She refused therapy with corticosteroids for IPH. She developed weight loss and dyspnea without iron-deficiency 
Table 1. Clinical Course of Pulmonary Function, esPAP and BMI.

\begin{tabular}{lcccc}
\hline & $\begin{array}{c}\text { Initial visit } \\
\text { (X year) }\end{array}$ & $\begin{array}{c}\text { Surgical lung biopsy } \\
\text { (X+3years) }\end{array}$ & $\begin{array}{c}\text { Lung transplantation } \\
\text { (X+5years) }\end{array}$ \\
\hline VC (L) & 1.6 & 1.31 & 0.75 & 1.21 \\
\%VC (\%) & 63.1 & 53.5 & 29.4 & 46.7 \\
FEV1 (L) & 0.6 & 0.4 & 0.3 & 1.12 \\
\%FEV1 (\%) & 30.6 & 19.9 & 16.9 & 55.2 \\
FEV1 (\%) & 48.9 & 40.8 & 44.2 & 95.7 \\
RV (L) & 1.8 & & 1.8 & \\
\%RV $(\%)$ & 133.8 & & 132.4 & \\
RV/TLC $(\%)$ & 53.2 & & 68.4 & \\
DLCO (mL/min/mmHg) & 11.3 & & 7.8 & 9.63 \\
\%DLCO $(\%)$ & 79.7 & & 61.5 & 46.3 \\
esPAP $(\mathrm{mmHg})$ & 16 & 26 & 44 & 21 \\
BMI (kg/m $\left.{ }^{2}\right)$ & 16.9 & 16.6 & 15.1 & 15.5 \\
\hline
\end{tabular}

VC: vital capacity, FEV1: forced expiratory volume \% in 1 second, RV: residual volume, TLC: total lung capacity, DLCO: diffusing capacity for carbon monoxide, esPAP: estimated systolic pulmonary artery pressure, BMI: body mass index
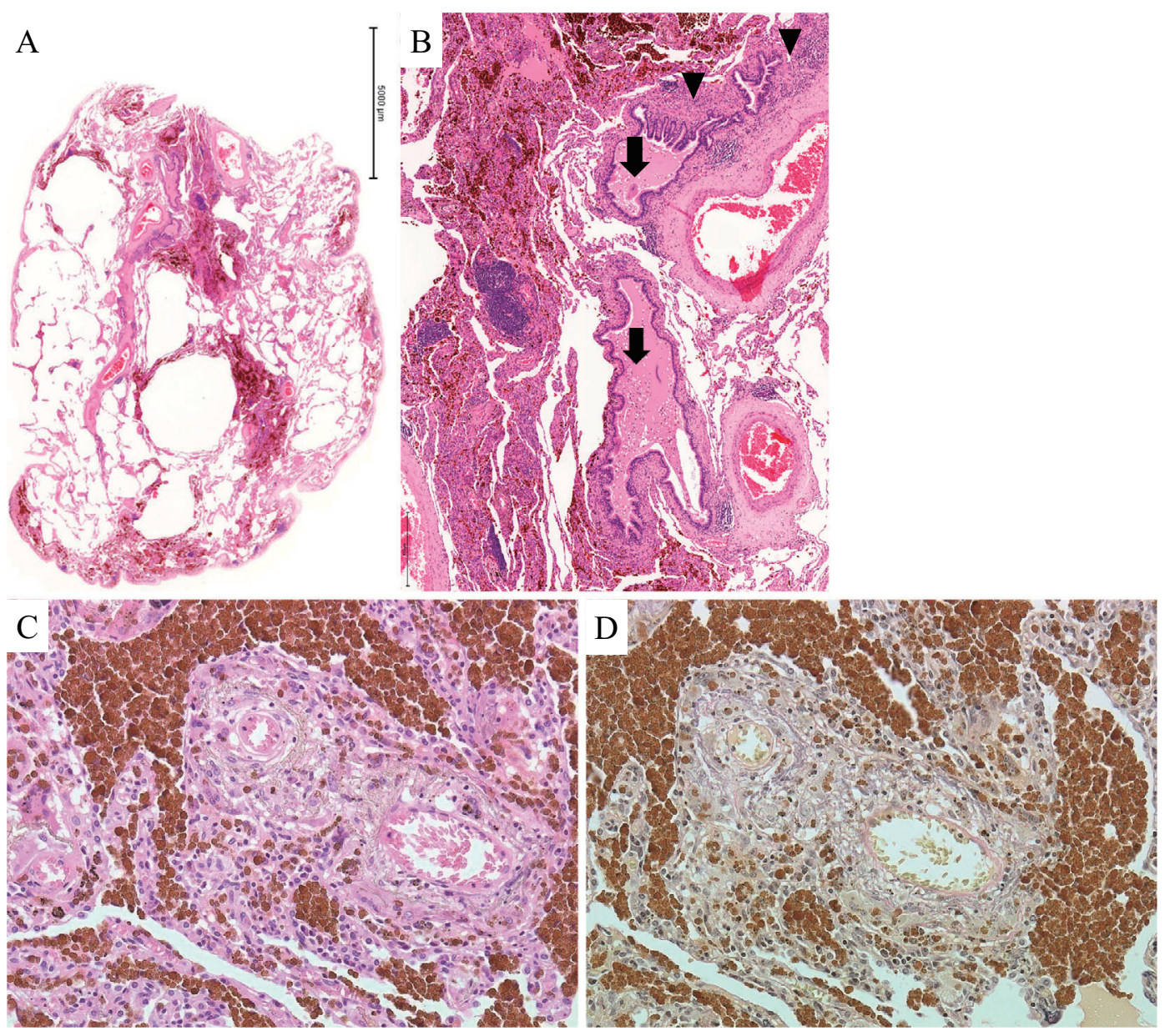

Figure 2. (A) The video-assisted thoracoscopic surgery specimen showed emphysematous change in addition to hemosiderin deposition in the peribronchial alveolar space and alveolar septum. (B) Terminal bronchioles associated with hemosiderosis lesions were dilated with the accumulation of mucus (arrow) and lymphocyte infiltration into the mucosa (arrowhead). (C, D) Hemosiderin and foreign body giant cells were deposited in the vessel walls, and elastic fibers of the vessel walls of the same lesion were obscured. However, vasculitis was not detected. (C) Hematoxylin and Eosin staining; (D) Elastica van Gieson staining. 


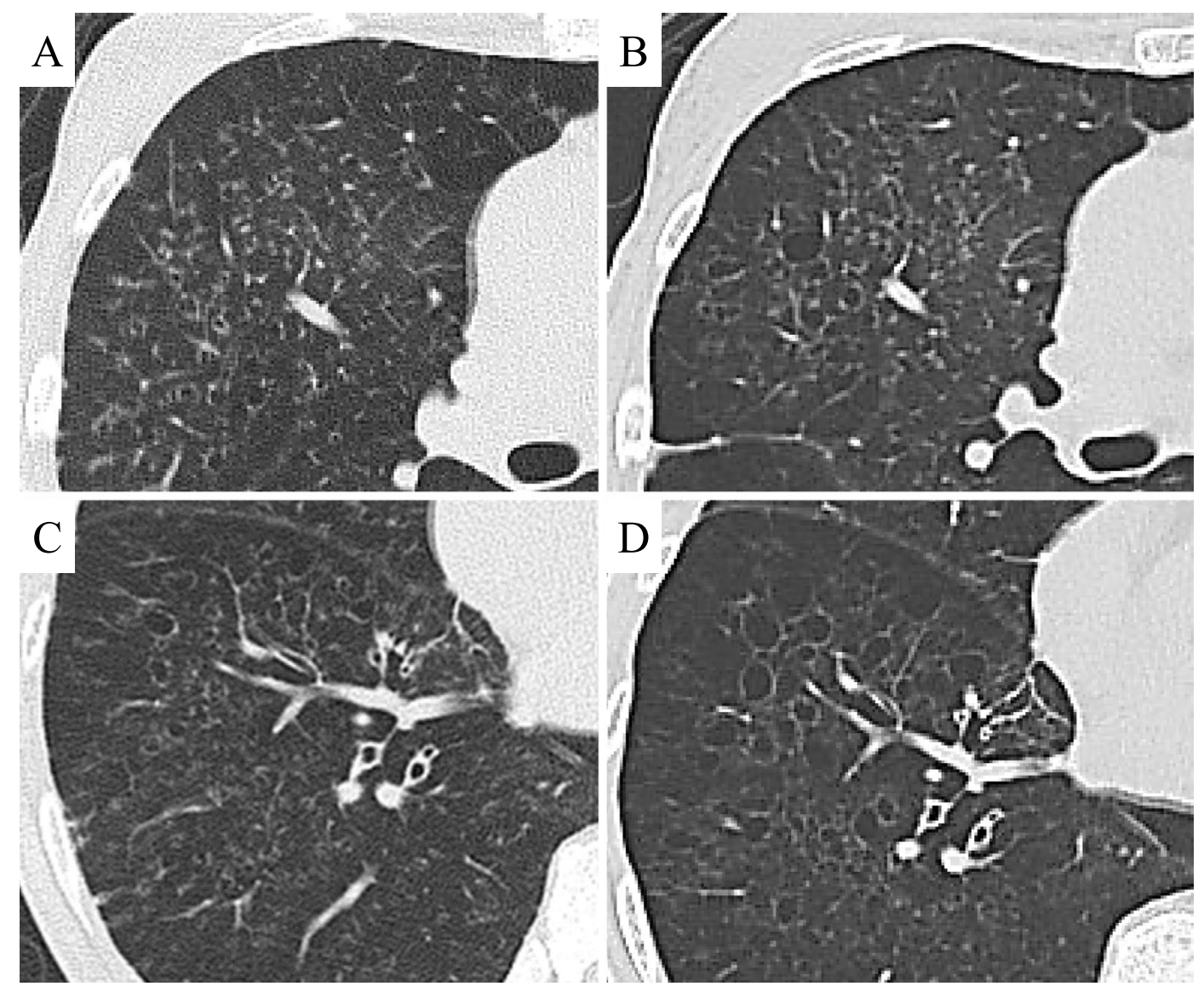

Figure 3. The comparison of computed tomography images between the initial visit and 5 years after the initial visit (before lung transplantation). The centrilobular distribution of small nodules in the upper lobes had progressed to emphysematous change and ectasic bronchiole over the five-year period. (A) Initial visit. (B) Five years later. The cysts and emphysema of the lower lobes showed an increased diameter, distorting the lung architecture. (C) Initial visit. (D) Five years later.

anemia, hemoptysis, and infiltration on chest images. Her lung function deteriorated progressively, resulting in hypercapnia and pulmonary hypertension (Table 1).

Over the five years from the initial visit, chest CT revealed that most of the small nodular infiltration of the upper lobes had progressed to cysts and ectasic bronchioles, whereas the cysts and emphysema of the lower lobes had increased in diameter, distorting the lung architecture (Fig. 3A, C show findings at the initial visit, B and D show findings five years after the initial visit, before lung transplantation). Although she received respiratory rehabilitation and oxygen therapy with non-invasive positive-pressure ventilation, her symptoms persisted. At five years after the initial visit, living-donor lung transplantation from her husband and daughter was performed because of an irreversible medical condition associated with pulmonary hypertension. The right lung received a transplant from her husband, and the left lung received a transplant from her daughter. The isolated lungs revealed peribronchial hemosiderosis with emphysematous change similar to that observed in the previous VATS specimen.

\section{Discussion}

Although IPH is suspected to involve immunological mechanisms, these processes remain unclear $(6,7)$. IPH commonly develops in childhood, and the onset of the disease in adulthood is relatively rare. Nevertheless, in recent years, cases of adult-onset IPH have been documented, revealing differences compared with pediatric cases $(8,9)$.

Our case exhibited some differences from previously reported cases of IPH in terms of the clinical course, as well as imaging and pathological findings (Table 2). First, the patient did not present the classic IPH triad hemoptysis, iron deficiency anemia, and infiltration on chest images. However, she eventually underwent living-donor lung transplantation due to type 2 respiratory failure and pulmonary hypertension in association with disease progression. Table 2 shows a review of the past 10 years of Japanese IPH patients. Miwa et al. and Chen et al. reported that, in contrast to pediatric cases, complication with iron deficiency anemia is rare, and treatment with steroids is more effective in adult-onset cases $(8,9)$. This type of treatment in adult patients is associated with fewer relapses and a good progno- 
Table 2. The Cases of Adult Onset IPH in Japan during Past 10 Years.

\begin{tabular}{|c|c|c|c|c|c|c|c|c|c|c|c|}
\hline \multirow[b]{2}{*}{ No } & \multirow[b]{2}{*}{ Reference } & \multirow{2}{*}{$\begin{array}{l}\text { Age/ } \\
\text { sex }\end{array}$} & \multirow{2}{*}{$\begin{array}{c}\text { Smoking } \\
\text { history }\end{array}$} & \multirow[b]{2}{*}{ Hemoptysis } & \multirow[b]{2}{*}{ Anemia } & \multicolumn{4}{|c|}{ CT findings } & \multirow[b]{2}{*}{ Treatment } & \multirow[b]{2}{*}{ Outcome } \\
\hline & & & & & & Consolidation & GGO & $\begin{array}{l}\text { Diffuse } \\
\text { nodules }\end{array}$ & Emphysema & & \\
\hline 1 & 18) & $62 / \mathrm{F}$ & Never & + & + & - & - & - & - & Steroids & Remission \\
\hline 2 & 19) & $31 / \mathrm{F}$ & Never & - & + & + & + & - & - & Steroids & Remission \\
\hline 3 & 15) & $34 / \mathrm{M}$ & Former & - & - & - & + & + & + & Steroids & ND \\
\hline 4 & 5) & 48/M & Current & + & - & - & + & - & - & Steroids & Remission \\
\hline 5 & 5) & $70 / \mathrm{M}$ & Former & + & - & - & + & - & - & Steroids & Remission \\
\hline 6 & 5) & $61 / \mathrm{M}$ & Former & + & - & + & + & - & - & Steroids & Remission \\
\hline 7 & 5) & $52 / \mathrm{M}$ & Former & + & + & + & + & - & - & Steroids & Remission \\
\hline 8 & 5) & 38/M & Current & + & - & + & + & - & - & - & Remission \\
\hline 9 & 5) & $43 / \mathrm{M}$ & Current & + & - & - & + & - & - & - & Remission \\
\hline 10 & 5) & $52 / \mathrm{M}$ & Former & + & - & + & + & - & - & - & Remission \\
\hline 11 & 5) & $76 / \mathrm{M}$ & Former & - & + & + & + & - & - & Steroids & Remission \\
\hline 12 & 5) & $65 / \mathrm{M}$ & Former & + & + & + & + & - & - & Steroids & Remission \\
\hline \multicolumn{2}{|c|}{ Our case } & $52 / \mathrm{F}$ & Never & - & - & - & - & + & + & Transplantation & Remission \\
\hline
\end{tabular}

GGO: ground glass opacity, M: male, F: female, ND: not described

sis (8) (Table 2). To our knowledge, only one previous case of IPH that underwent lung transplantation has been reported; the case relapsed several years after transplantation (10). In our case, five years following the lung transplantation, the patient had no relapse of IPH, although restrictive allograft syndrome coexisted in the transplanted lung four years after lung transplantation. To date, the efficacy of lung transplantation remains controversial because the number of cases is too small to adequately evaluate. However, in our case, lung transplantation helped the patient free herself from progressive disease and improved her dyspnea and activity compared with before transplantation.

The currently available literature on imaging and histopathological findings of IPH is relatively scarce, and differing results are reported, depending on the clinical phase. At the acute phase after bleeding, blood cells and hemosiderin infiltrate the alveolar space and alveolar septa, and chest CT shows a reticular shadow (ground glass shadow) and/or infiltration. Subsequently, these shadows are absorbed and disappear through treatment and the natural course, and damage to the existing lung structure is limited. Centrilobular small nodules on $\mathrm{CT}$ in the subacute and chronic phase indicate aggregation of hemosiderin and hemosiderin-laden macrophages around the bronchi $(3,4,11,12)$. When repeated bleeding becomes chronic, the lung structure may be modified. This leads to the development of honeycomb lung and non-specific fibrosis $(4,13,14)$.

As reported by several researchers, CT in our case revealed centrilobular small nodules, bronchial dilation, and emphysematous change (Table 2). The centrilobular small nodules in our case had not disappeared after a short period, instead progressing to cysts and bronchiole dilation over several years. Cheah et al. reported cases in which more than several years had passed since the first instance of hemoptysis, and small nodules remained on CT scans despite the absence of hemoptysis (11).
Complication of IPH and emphysematous change is very rare. Ikeda et al. reported that IPH is complicated with emphysematous change (15). In that case, they suspected that the bleeding might have caused destruction of the existing lung structure, given the hemosiderin deposition in the destructive alveolar elastic fibers. Stainer et al. reported that anti-neutrophil cytoplasmic antibody (ANCA) positivity and peribronchovascular emphysema developed with repeated hemoptysis in patients initially diagnosed with IPH. They speculated that proteolytic enzymes and free oxygen radicals released by vasculitis destroyed the alveolar walls and caused emphysema (16). However, in our case, although the involvement of noxious insults associated with hemorrhaging cannot be ruled out, the formation of emphysema is considered to involve bronchioles strictured by hemosiderosis potentially causing air trapping, which expands to more peripheral bronchioles and alveoli, thereby eventually leading to emphysematous change. To our knowledge, this finding had not been previously reported.

In addition, the lung specimen revealed the vulnerability and obscurity of the elastic fibers in the alveolar septa of the emphysema and small blood vessel at the site of hemosiderosis. The abnormalities of the elastic fibers were distributed around the site of peribronchial hemosiderosis. As noted above, we consider the stricture of the bronchioles induced by hemosiderosis to have expanded to peripheral bronchioles and emphysematous change; however, abnormality of the elastic fibers itself may lead to the development of emphysematous change. The etiology of vascular type Ehlers-Danlos syndrome is a deficiency in the production of type III collagen. This can cause alveolar hemorrhaging due to the destruction of pulmonary blood vessels and cyst formation due to the rupture of the stroma (17). Therefore, it is necessary to distinguish vascular-type EhlersDanlos syndrome from IPH. In our case, the presence of pulmonary hemorrhaging only (i.e., no extra-pulmonary ab- 
normality, such as in the skin and joints) ruled out vasculartype Ehlers-Danlos syndrome. However, incomplete EhlersDanlos syndrome or undiagnosed connective tissue fragility disease may have led to the development of peribronchial hemosiderosis and emphysematous change.

Our case was conclusively diagnosed with IPH, mainly based on the histopathological findings and an exclusion diagnosis. However, the patient exhibited differences from previous reports with regard to clinical symptoms, imaging and histopathological findings, and the clinical course. Therefore, we must exercise caution when diagnosing IPH. This was an interesting case, as the imaging and pathological findings were able to be compared throughout the clinical progression.

Written informed consent for the publication of the clinical details and images was provided by the patient.

The authors state that they have no Conflict of Interest (COI).

\section{Acknowledgement}

The authors greatly thank Dr. Harumi Itoh of University of Fukui Biomedical Imaging Research Center and Dr. Takashi Ogura of the Department of Respiratory Medicine and Kanagawa Cardiovascular and Respiratory Center for their general support in the diagnosis and treatment.

\section{References}

1. Waldenstrom J. Pulmonary bleedings or hemosiderosis pulmonuma new clinical diagnosis. Acta Radiol 25: 149-162, 1944.

2. Morgan PG, Turner-Warwick M. Pulmonary haemosiderosis and pulmonary haemorrhage. Br J Dis Chest 75: 225-242, 1981.

3. Milman N, Pedersen FM. Idiopathic pulmonary haemosiderosis. Epidemiology, pathogenic aspects and diagnosis. Respir Med 92: 902-907, 1998.

4. Ioachimescu OC, Sieber S, Kotch A. Idiopathic pulmonary haemosiderosis revisited. Eur Respir J 24: 162-170, 2004.

5. Mukai Y, Agatsuma T, Ideura G. Early diagnosis of idiopathic pulmonary haemosiderosis: increased haemosiderin-laden macrophages in repeat bronchoscopy. Respirol Case Rep 6: e00304, 2018.

6. Heiner DC, Sears JW, Kniker WT. Multiple precipitins to cow's milk in chronic respiratory disease. A syndrome including poor growth, gastrointestinal symptoms, evidence of allergy, iron defi- ciency anemia, and pulmonary hemosiderosis. Am J Dis Child 103: 634-654, 1962.

7. Taytard J, Nathan N, de Blic J, et al. New insights into pediatric idiopathic pulmonary hemosiderosis: the French RespiRare ${ }^{\circledR}$ cohort. Orphanet J Rare Dis 8: 161, 2013.

8. Miwa S, Imokawa S, Kato M, et al. Prognosis in adult patients with idiopathic pulmonary hemosiderosis. Intern Med 50: 18031808, 2011.

9. Chen XY, Sun JM, Huang XJ. Idiopathic pulmonary hemosiderosis in adults: review of cases reported in the latest 15 years. Clin Respir J 11: 677-681, 2017.

10. Calabrese F, Giacometti C, Rea F, et al. Recurrence of idiopathic pulmonary hemosiderosis in a young adult patient after bilateral single-lung transplantation. Transplantation 74: 1643-1645, 2002.

11. Cheah FK, Sheppard MN, Hansell DM. Computed tomography of diffuse pulmonary haemorrhage with pathological correlation. Clin Radiol 48: 89-93, 1993.

12. Khorashadi L, Wu CC, Betancourt SL, Carter BW. Idiopathic pulmonary haemosiderosis: spectrum of thoracic imaging findings in the adult patient. Clin Rad 70: 459-465, 2015.

13. Harte S, McNicholas WT, Donnelly SC, Dodd JD. Honeycomb cysts in idiopathic pulmonary haemosiderosis: high-resolution CT appearances in two adults. Br J Radiol 81: e295-e298, 2008.

14. Buschman DL, Ballard R. Progressive massive fibrosis associated with idiopathic pulmonary hemosiderosis. Chest 104: 293-295, 1993.

15. Ikeda $S$, Hagiwara E, Nakazawa $A$, et al. An adult case of idiopathic pulmonary hemosiderosis with progressive structural destruction of pulmonary alveolus. Nihon Kokyuki Gakkai Zasshi 2: 354-358, 2013 (in Japanese, Abstract in English).

16. Stainer A, Rice A, Devaraj A, et al. Diffuse alveolar haemorrhage associated with subsequent development of ANCA positivity and emphysema in three young adults. BMC Pulm Med 19: 185, 2019.

17. Kawabata Y, Watanabe A, Yamaguchi S, et al. Pleuropulmonary pathology of vascular Ehlers-Danlos syndrome: spontaneous laceration, haematoma and fibrous nodules. Histopathology 56: 944950, 2010.

18. Harada N, Motegi M, Sato M, Ueno M, Shimizu Y. A case of idiopathic pulmonary hemosiderosis with recurrent episodes of small hemoptysis for thirty years. Jpn J Chest Dis 75: 786-793, 2016 (in Japanese, Abstract in English).

19. Nakano H, Inoue S, Igarashi A, Abe S, Shibata Y, Kubota I. A case of acute onset diffuse alveolar hemorrhaging due to idiopathic pulmonary hemosiderosis. Jpn J Chest Dis 73: 1112-1117, 2014 (in Japanese, Abstract in English).

The Internal Medicine is an Open Access journal distributed under the Creative Commons Attribution-NonCommercial-NoDerivatives 4.0 International License. To view the details of this license, please visit (https://creativecommons.org/licenses/ by-nc-nd/4.0/).

(C) 2021 The Japanese Society of Internal Medicine

Intern Med 60: 117-122, 2021 